\title{
Acupuncture for controlling the gag reflex during upper alginate impression
}

The use of acupuncture in controlling the gag reflex in patients requiring an upper alginate impression: an audit P. Rosted, M. Bundgaard, J. Fiske and A. M. L. Pedersen Br Dent J 2006; 201: 721-725

\section{Background}

A pronounced gag reflex (GR) can be a problem to both the acceptance and delivery of dental treatment. Despite a range of management strategies, some patients cannot accept even simple dental treatment. The aim of this study was to evaluate the use of acupuncture point CV-24 in controlling a profound gag reflex during dental treatment requiring an upper alginate impression.

\section{Method}

Members of the British Dental Acupuncture Society were invited to take part in an audit of the role of acupuncture point CV-24 in controlling the gag reflex. They were issued with patient inclusion criteria, a standardised procedure instruction sheet and a recording form. All patients fulfilling the inclusion criteria had an upper dental alginate impression taken (or an attempt made at it) before acupuncture, and a second upper alginate impression taken immediately after acupuncture of point CV-24. The GR assessment was undertaken prior to insertion of the acupuncture needle using the Gagging Severity Index (GSI); and after the acupuncture and impression taking using the Gagging Prevention Index (GPI). Both the GSI and GPI were recorded at three stages of the dental impression taking procedure, ie, when the empty impression tray was tried in the mouth, when the loaded tray was inserted into the mouth, and on completion of the impression taking. Results

Twenty-one dentists submitted 64 case reports of which 37 fulfilled the inclusion criteria. Prior to acupuncture all 37 patients (20 females and 17 males with a mean age of 46.8 years) were unable to accept the impression taking. After acupuncture of point CV-24, an improvement of between $51-55 \%$ (mean 53\%) for the three stages of impression taking was noticed. Thirty patients (81\%) were able to accept the impression taking, whereas seven (19\%) remained unable to tolerate the procedure. Assessed by the GSI and GPI, there was a significant decrease in GR scores at all three stages of the impression taking procedure (median 3 vs $1 ; 4$ vs $2 ; 4$ vs $2 ; p<0.0001$ ). Thus before acupuncture, the patients had moderate to severe GR and after acupuncture the GR had reduced to a level which only complicated dental treatment slightly.

\section{Conclusion}

Our results indicate that acupuncture of point CV-24 is an effective method of controlling severe GR during dental treatment including impression taking. However, the results of the current audit need to be tested in a randomised controlled study in order to substantiate the effectiveness of this method.

\section{IN BRIEF}

- Acupuncture can be timesaving in patients with severe gag reflex.

- Acupuncture has a nearly instant effect in controlling the gag reflex in eight out of ten patients.

- Acupuncture can reduce the discomfort for patients suffering from a severe gag reflex during dental treatment.

\section{COMMENT}

The gag reflex has profound impact on the provision of dental care for patients who are affected. The reflex interferes with the ability of the dental practitioner to diagnose, radiograph, and provide treatment for dental patients, and leads to increased levels of stress suffered by patients during treatment sessions. Treatment plans may well be compromised by the need to limit the impact of the reflex, and in some cases unnecessary levels of intervention may be required, eg general anaesthesia.

In addition the reflex can be so troublesome that it inhibits patient attendance for treatment, and induces anxiety related to dental treatment in certain patients. In severe cases patients actively avoid dental treatment. Therefore any appropriate method of controlling the reflex is welcomed. Approaches range from distraction therapy to general anaesthesia, but may carry significant morbidity.

Acupuncture has been proposed as a method of controlling the gag reflex. ${ }^{1,2}$ Acupuncture offers a rapid, simple, cheap, and low morbidity method of reliably controlling the gag reflex, even in those patients who struggle with treatment when sedated. ${ }^{1}$ There are a number of potential acupuncture points that may be employed to control the gag reflex, and in this paper a single acupuncture point is used, Conception Vessel 24, which suggests a significant level of reproducibility. This paper takes audit material generated by members of the British Dental Acupuncture Society with patients requiring upper alginate impressions, a potent stimulus for the gag reflex. Although not a placebo controlled trial, the results clearly demonstrate evidence of efficacy of acupuncture in controlling the gag reflex, with an effectiveness much greater than that which might be expected with a placebo response.

We often comment that there are few really new ideas. Acupuncture is a technique that is thousands of years old, yet has been undergoing a renaissance in the west over the last twenty years or so, and has found significant favour in the medical profession and the professions allied to medicine. The management of the gag reflex is an excellent starting position for the dental profession to also embrace this technique to the benefit of our patients.

T. Thayer, Senior Dental Officer/Specialist in Surgical Dentistry, Central Cheshire PCT

1. Fiske J, Dickenson $\mathrm{C} M$. The role of acupuncture in controlling the gagging reflex using a review of ten cases. Br Dent J 2001; 190: 611-613.

2. Lu D P, Lu G P, Reed J F. Acupuncture/acupressure to treat gagging dental patients: a clinical study of anti-gagging effects. Gen Dent 2000; 48: 446-452.

DOI: $10.1038 /$ sj.bdj.4814307 